\title{
Construction of distributed computer test system of the ammunition warehouse based on the grid technology
}

\author{
Yukui Ding ${ }^{\mathrm{a}}$, Wensu Ji ${ }^{\mathrm{b} *}$, Haidan Wang ${ }^{\mathrm{a}}$, Huaizhi Zhang ${ }^{\mathrm{b}}$, Huiming $\mathrm{Li}^{\mathrm{b}}$, Quanmin $\mathrm{Xie}^{\mathrm{b}}$ \\ ${ }^{a}$ Ordnance Engineering College, Shijiazhuang 050003, China \\ ${ }^{\mathrm{b}}$ Wuhan Ordnance N.C.O Academy of PLA, Wuhan 430075, China \\ *Corresponding author. Telephone number:18602724022. Email address: jiwensu@sina.com
}

\begin{abstract}
Taking into account the requirements of the data processing and transmission of a large number of real-time monitoring information for the server hardware resources while establishing national ammunition warehouses, a national ammunition warehouse distributed test system is established based on the grid technology. The system has expandable calculation ability, high calculation efficiency and low cost with preferable application prospects.
\end{abstract}

\section{Keywords-grid technology; node;parallel computing}

\section{INTRODUCTION}

It is of practical significance for improving our ammunition support capacity to construct environment information and ammunition quality monitoring system in the national ammunition warehouse. To grasp the major ammunition warehouse information timely and accurately ,we need to real-timely monitor warehouse temperature, humidity and other environmental information, video, audio and other site information. Meanwhile, ammunition quality and other related information still need monitoring timely to ensure the inventory ammunition performance. In order to meet vast monitoring information processing and real-time data transmission, a higher demand for servers and other hardware resources should be set. It becomes a brand-new subject to construct a set of high-efficiency, low-cost distributed test system combining the current domestic and international advanced computer network and parallel computing technology.

In recent years, grid computing has become a hotspot of the computer research field [1-4]. The technology can provide a new way of shared computer resources, and it is able to make these resources work collaboratively in a heterogeneous, distributed and dynamic environment. Many industries have begun to apply the grid technology, with which different testing techniques would be integrated to establish a more modern grid. Therefore, the author makes an attempt to build a national ammunition depots distributed test system based on grid technology. The measured data show that good results and the expected objectives were obtained.

\section{GRID TECHNOLOGY}

VMG uses Globus ${ }^{\circledR}$ Toolkit 4 (GT4) to carry out task management and authority management [5]. Globus ${ }^{\circledR}$ Toolkit is the foundation for building grid components to share computing resources, databases, and other toolkit safely across the company, institution, geographic restrictions, which includes software services and libraries on resource monitoring, discovery, management, and security certification. VMG uses "Condor" to build computing pool, including a large number of computing nodes. Condor is load management system that makes compute-intensive as customization, which provides job queue mechanism, job distribution mechanisms, priority scheme, resource monitoring mechanism and the management mechanism. Meanwhile, Condor allows users to use the computer to build a computing pool, and the job will automatically be distributed to the calculation nodes in the computing pool [6].

\section{MULTITASKING SUBMIT MECHANISM DESIGN}

According to the actual network environment of the current national ammunition depots, the design divided VMG into three layers, as shown in Fig.1. A multilayer submitting mechanism is designed to achieve operation at different levels in the submission, the security of data transmission as well as automatic segmentation and calculation job.

The first layer consists of a national control center and data center. National control center receives tasks submitted from data centers of the provincial ammunition warehouse, and automatically distributes job information to the appropriate provincial control center.

The second layer contains a number of provincial control centers, recieving the task submitted from the national control center. Subsequently, the computing centers at the provincial level gather the related data from the national data center such as ammunition depots environment, ammunition quality performance and so on. In order to make better use of computing power provided by the computing pool, the tasks here will be divided into a number of small-scale operations. All small assignments will be given out into the job queue, and eventually submitted to the provincial computing center.

The third layer contains a number of provincial-level computing centers, and computing centers in the provinces configure a large number of computing nodes. Each computer distributed in the ammunition warehouses assumes the function of the minimum computing nodes. Compute nodes perform respectively various small-scale operations. Task scheduling will make the entire system in state of load balancing. When provincial calculation node finishes the job, 
the calculation results will return to the provincial control node.

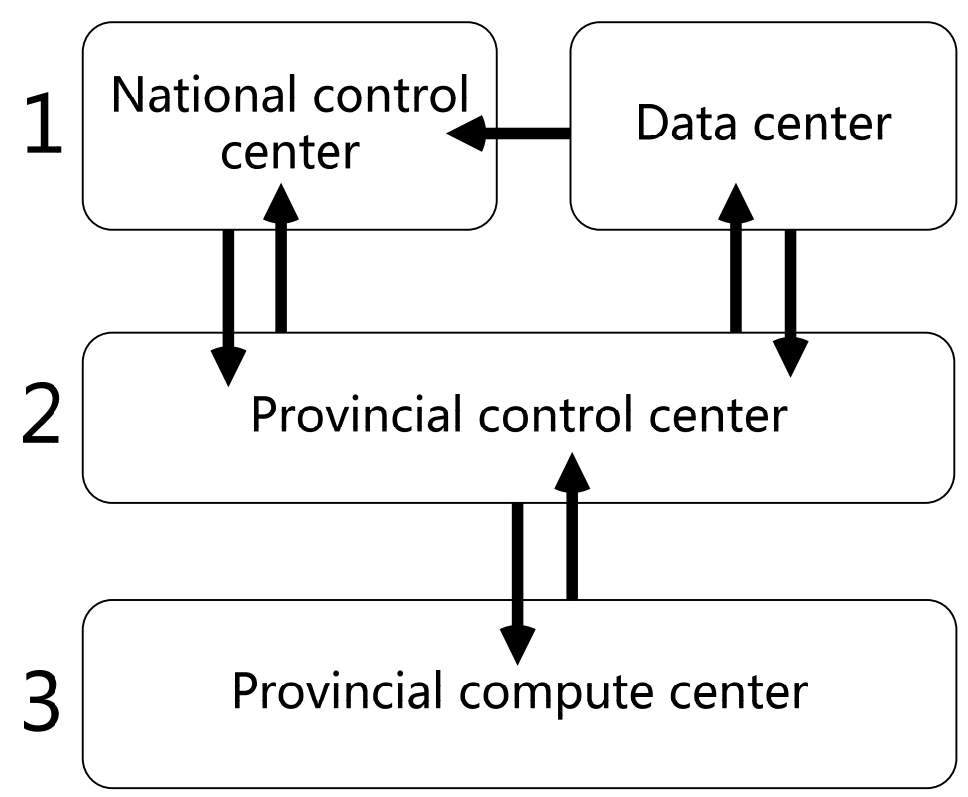

Fig.1 Three-layer structure of the grid

\section{FILE TRANSFER MECHANISM AND GRID} PERFORMANCE OPTIMIZATION

\section{A. File Transfer Mechanism by Condor}

VMG uses condor to set up calculation node pool. However, condor file transmission mechanism has its defects. In some special cases, the program running in the computing pool is quite huge, or all calculation nodes share a huge input file. In these cases, all the computing nodes must obtain the same file from the central management node or file server. Although it is possible to reduce the waste of network bandwidth by using the Network File System, the problem has not been fundamentally resolved.

For example, when we use a computing pool to compress a number GB of video files, all of the nodes will obtain the video files from the center management server, as shown in Fig.2 (a). Obviously, the export bandwidth of the center management server will become a bottleneck. When calculation pool is large enough, the need to transfer the amount of data will be great. Therefore, the time of the system spent transferring data will not be accepted.

\section{B. Using P2P System for File Transfer}

VMG use a P2P file system WheelFS to realize P2P file transfer.

When we do not use the peer-to-peer file system, Condor obtains data file from one node, as shown in Fig.2 (a) below. Obviously, time complexity for the file transfer is $\mathrm{O}(\mathrm{N})$.

In the traditional network file system, when using NFS, although all clients get a file from one server, but they can directly and logically "visit" files, and do not need to wait for the file transmission finished. The time complexity is also $\mathrm{O}(\mathrm{N})$, but the whole process can be accelerated.

It is desirable that the performance of data transmission can be enhanced, as shown in Fig.2 (b), by using a peer-to-peer file system. On one hand, all of client nodes sequentially read a file, which is the worst case. On the other hand, which is more often, one client makes access to the cache file block from a number of the other clients at the same time, and the time complexity is $\mathrm{O}(\log N)$. 

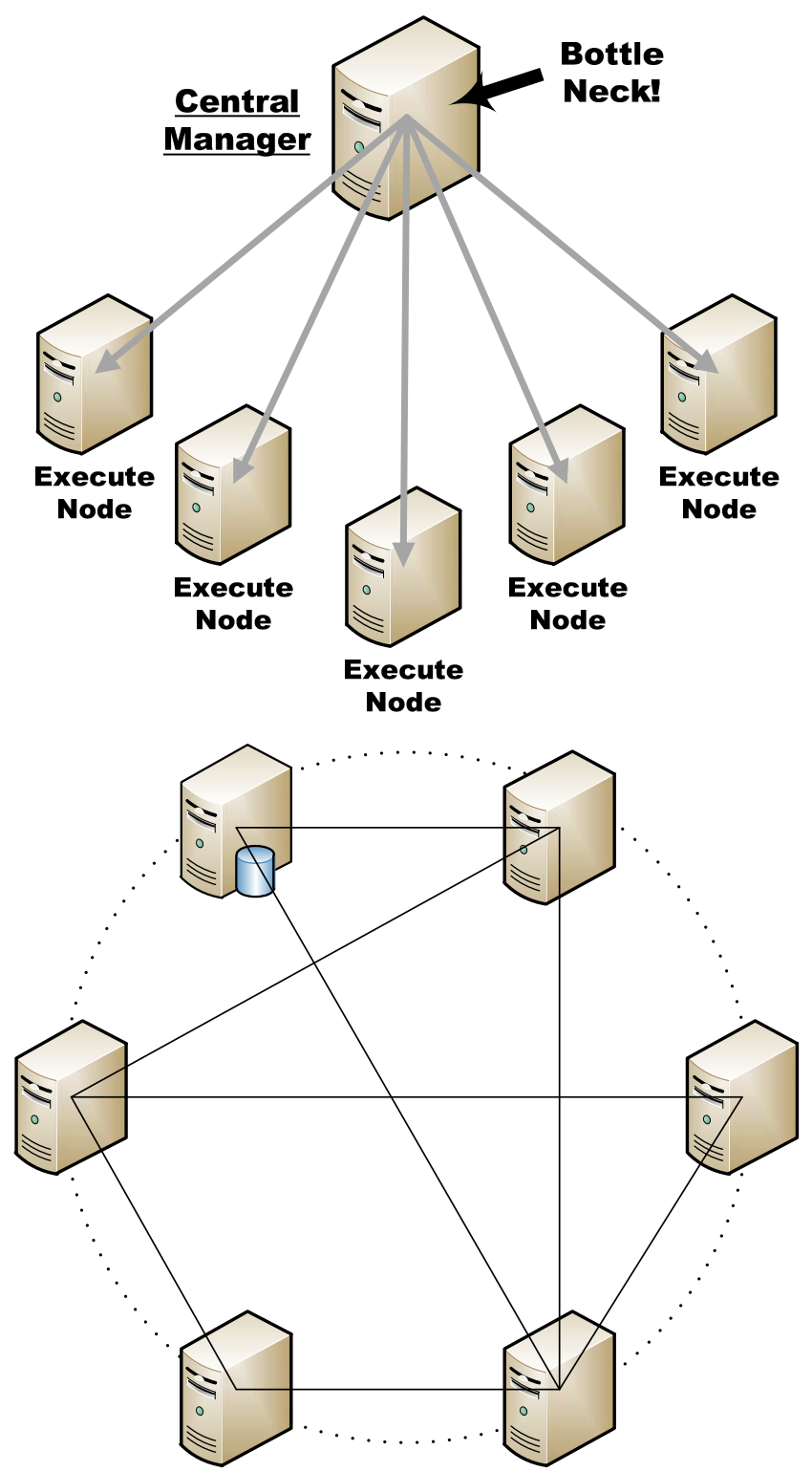

Fig.2 (a) File transfer example of Condor

(b) File transfer example of P2P network

\section{Grid Performance Test}

We test the read performance of computing pool file mounting 'WheelFS'. All calculation nodes in the computing pool randomly read a data file obtained by real-time monitoring from some ammunition warehouse. For comparative analysis, using NFS and not using any of the distributed file system has been tested. We have read five documents in the test, ranging in size from $21 \mathrm{MB}$ to $3.2 \mathrm{~GB}$.
As shown in Fig.3, the file transfer time is the longest, in case of not using a distributed file system, and it can be found that the transmission time increase sharply with increasing size of the file. However, time consumption dropped significantly in case of using NFS, mainly because that the compute nodes do not need to wait for the entire file transfer to have been finished before starting a calculation. Finally, in the case of using $\mathrm{P} 2 \mathrm{P}$ file system, the time consumption is minimum, and as the file size increases, the advantage of P2P file system is more and more obvious. 


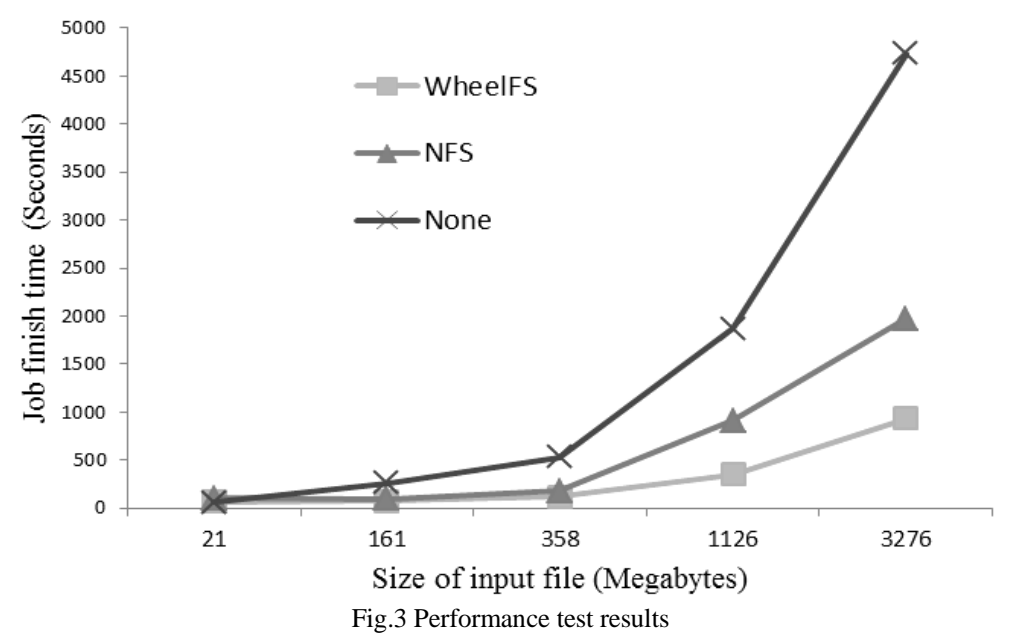

\section{CONCLUSION}

The paper presents the design of the multilayer submitting mechanism to make VMG run in actual hierarchical network, which use a number of low-cost PC distributed in the provincial ammunition warehouse to form the grid as to improve computing speed. Furthermore, the performance of the Condor computing pool is improved dramatically through the mount P2P file system. The simulation results show that $\mathrm{P} 2 \mathrm{P}$ file system can greatly improve the efficiency of the job processing. Especially when sharing huge input file, P2P technology can greatly reduce the processing time of the job, which shows that a set of low-cost, high-efficiency ammunition warehouse distributed test system is constructed successfully.

\section{ACKNOWLEDGMENT}

The author thanks Quanmin Xie and Huiming Li from Wuhan Ordnance N.C.O Academy of PLA for many useful discussion at the early stage of this work.

\section{REFERENCES}

[1] WANG Xiao-jin,PENG Jiong,YANG Ling and CHEN Jin-nan, "Numerical Calculation of Solid-liquid Mixing Time in the Planetary Mixer,” Computers and Applied Chemistry, vol. 03, 2012, pp. 47-53.

[2] GAN Liang, YU Li-li, LI Run-heng, JIA Yan and JIN Xin, “A Reverse Dominant Point Set Based Algorithm for Top-k Queries in DSMS," Computer Engineering \& Science, vol. 06, 2012, pp. 124-130.

[3] LU Jin-guo, LIANG Zhong-gang, ZHOU Yi-mei and WU Fang-liang, "Effect of Turbulence Model and Mesh Distribution on Numerical Computation of Rotary Hydrodynamics of Underwater Vehicle,” Computer Aided Engineering, vol. 03, 2012, pp. 86-92.

[4] ZHU Ting-ru, PENG Xiao-dong, WANG Gang, ZHAO Xiao-ming and TANG Chang-jian, "Two Dimensional High-order CIP Numerical Method and Applications," Nuclear Fusion and Plasma Physics, vol. 02, 2012, pp. 92-98.

[5] WANG Yong-zhi, SHENG Ye-hua, ZHOU Liang-chen, CHEN Jian-zhong and LIANG Yan, "Generation Method of Three-dimensional Computing Model of Wall Rock of Salt Caverns Supported by 3D GIS,” Rock and Soil Mechanics, vol. 05, 2012, pp. 103-108.

[6] FU Yin-jin, XIAO Nong, LIU Fang and BAO Xian-qiang, "Deduplication Based Storage Optimization Technique for Virtual Desktop,” Journal of Computer Research and Development, vol. S1, 2012, pp. 65-70. 